\title{
High resolution kinetic beam schemes in generalized coordinates for ideal quantum gas dynamics
}

\author{
Yu-Hsin Shi, J.C. Huang, J.Y. Yang * \\ Institute of Applied Mechanics, National Taiwan University, 1, Sec. 4, Roosevelt Road, Tapei, Taiwan 10764, Taiwan
}

Received 17 August 2005; received in revised form 16 June 2006; accepted 7 August 2006

Available online 18 September 2006

\begin{abstract}
A class of high resolution kinetic beam schemes in multiple space dimensions in general coordinates system for the ideal quantum gas is presented for the computation of quantum gas dynamical flows. The kinetic Boltzmann equation approach is adopted and the local equilibrium quantum statistics distribution is assumed. High-order accurate methods using essentially non-oscillatory interpolation concept are constructed. Computations of shock wave diffraction by a circular cylinder in an ideal quantum gas are conducted to illustrate the present method. The present method provides a viable means to explore various practical ideal quantum gas flows.
\end{abstract}

(c) 2006 Elsevier Inc. All rights reserved.

Keywords: Ideal quantum gas; Quantum gas dynamics; Beam scheme; High resolution scheme; General coordinates

\section{Introduction}

The ideal classical gas dynamics can be described by the local equilibrium Maxwell-Boltzmann distribution which corresponds to the lowest order solution of the classical Boltzmann equation [1]. The conservation laws based on the Maxwell-Boltzmann distribution is the well known Euler equations of gas dynamics. Similar to the classical Boltzmann equation, a quantum Boltzmann equation for transport phenomenon can be developed for fermions and bosons, see [2,3]. The Chapman-Enskog procedure has been generalized for quantum gases in [2] to obtain the expressions for the transport coefficients such as shear viscosity and thermal conductivity. More recent works on the derivation of hydrodynamic equations of a trapped dilute Bose gas based on quantum Boltzmann equation using Chapman-Enskog procedure can be found in $[4,5]$.

In the past three decades, various Euler equations solvers have been constructed for aerodynamic and gas dynamical flow problems, particularly, involving shock waves, see [6,7]. Most methods are based on the macroscopic continuum description and the hyperbolic conservation law concept and characteristic information have been heavily implemented. In the mean time, Euler solvers based on the microscopic kinetic description using

\footnotetext{
* Corresponding author. Tel.: +88623366 5636; fax: +886223629290.

E-mail address: yangjy@iam.ntu.edu.tw (J.Y. Yang).
} 
Maxwell-Boltzmann distribution have been devised concurrently, for example, the beam scheme of Sanders and Prendergast [8], the equilibrium particle simulation method of Pullin [9], the method of Reitz [10] and the kinetic flux vector splitting method of Deshpande [11]. Basically, almost all the original Euler methods are of first-order accuracy and are too diffusive to represent flow structures. Normally, high-order methods can be devised for those basic methods to reduce the numerical diffusion, in particular, the so-called high resolution schemes with some flux limiters. The above mentioned gas-kinetic Euler solvers are also of first-order accuracy in space and possess some upwinding properties, i.e., in accordance with the characteristic wave propagation property and capture the gas dynamical discontinuities correctly although rather diffusively. Normally, high-order methods can be devised for the basic first-order methods to yield good computational tools for practical problems.

Beyond the kinetic schemes for the zeroth-order solution of the Boltzmann equations, there are several kinetic numerical methods for higher-order extensions to Navier-Stokes equations have been developed, for examples, Prendergast and Xu [12,13], Chou and Baganoff [14] and Ohwada [15]. However, they rely heavily on the BGK-type models [16] for treating the collision term. In this work, we shall confine ourself to the kinetic methods for the zeroth-order equilibrium limit solution nevertheless of the quantum Boltzmann equation.

In [8], Sanders and Prendergast presented an interesting explicit scheme, which they called the beam scheme, for solving the equilibrium limit of the classical Boltzmann equation. The derivation was based on the local thermodynamic equilibrium Maxwell-Boltzmann distribution and resulted a novel method for solving the transport processes governed by the Euler equations of Newtonian gas dynamics. Later in [17], the concept of beam scheme of Sanders and Prendergast was successfully extended to relativistic Boltzmann transport equation based on the Jüttner distribution. In the beam scheme, a presumed local thermodynamic equilibrium distribution function is approximated by several discrete Dirac delta functions or discrete beams of particles in each cell. These beams are permitted to move over a time step transporting mass, momentum and energy into adjacent cells. The motion of each beam is followed to first-order accuracy. The transport is taken into account to determine the new mass, momentum, and energy in each cell; and these macroscopic moments are used to describe the new local equilibrium distribution for each cell. The entire process is then repeated and advanced to the next time step. The choice of the size of the time step is dictated by the CourantFriedrich-Lewy stability condition that physically no beam of gas particles travels farther than one cell spacing in one time step. The beam scheme, although it is a particle scheme, has all the desirable features of modern characteristics-based wave propagating numerical methods, the so-called upwind shock-capturing methods for hyperbolic conservation laws of gas dynamics. The kinetic beam scheme turns out to have exactly the same form as the so-called flux vector splitting methods for the Euler equations of Newtonian gas dynamics $[18,19]$. This beam flux splitting method possesses an entropy-satisfying mechanism and can exclude expansion shocks. For further details and discussion of the beam scheme, see [8,17]. The basic beam scheme is firstorder accurate and a class of high-order ENO methods were also presented for the beam scheme [17].

In [20], the concept of classical beam scheme has been adopted to devise a numerical method for the computation of ideal quantum gas dynamics. Starting with the equilibrium limit of the quantum Boltzmann transport equation, one assumes the particles obey the Bose-Einstein or Fermi-Dirac statistics and the particles of the system are in the excited states. Similar to the ideal classical gas dynamics based on Maxwell-Boltzmann distribution, we can claim that the present method is for ideal quantum gas dynamics based on quantum statistics, namely the Bose-Einstein and Fermi-Dirac distributions. By approximating the quantum local equilibrium distribution by a superposition of several discrete Dirac delta functions (beams), each with suitable weight, such that the conserved macroscopic quantities such as number density (or mass density), momentum and energy defined by the moments of the distribution function are the same as those calculated by taking moments of the approximated discrete beams. The basic first-order quantum beam scheme has been derived for Bose-Einstein distribution and Fermi-Dirac distribution [20]. Formulations for one to three space dimensions in Cartesian coordinates have been provided. In this work, we shall adopt the ENO interpolation to the basic first-order beam method to yield a class of high-order beam splitting method for ideal quantum gas dynamics. Formulations in general coordinates system are also derived for treating general geometries. Probably, one of the gas dynamical problems which can exhibit most important features such as shock waves, contact surface and expansion waves and their nonlinear interaction is the unsteady shock wave diffraction by a finite body. In this work, numerical simulations of unsteady shock wave diffraction by a circular cylinder are carried out to test and illustrate the present beam-based methods. 
The paper is organized as following. We first briefly describe the elements of quantum Boltzmann transport equation and related formulation in two space dimensions in Section 2. In Section 3, the basic kinetic quantum beam scheme in two space dimensions is first described and then formulation in general coordinates system is given. In Section 4, implementation of high resolution methods is outlined. In Section 5, boundary and initial conditions for shock wave diffraction are described. In Section 6, numerical experiments of unsteady shock wave diffraction by a circular cylinder in both classical and nearly degenerate regimes are simulated. Lastly, some concluding remarks are given in Section 7. Formulation of the quantum beam scheme in three space dimensions in general coordinates system is included in Appendix A.

\section{Elements of quantum Boltzmann equation}

In this section, we briefly describe the elements of quantum Boltzmann transport equation appropriate for the development of present work. Following [2,3], we consider the quantum Boltzmann equation

$$
\left(\frac{\partial}{\partial t}+\frac{\vec{p}}{m} \cdot \nabla_{\vec{x}}-\nabla U(\vec{x}, t) \cdot \nabla_{\vec{p}}\right) f(\vec{p}, \vec{x}, t)=\left(\frac{\delta f}{\delta t}\right)_{\text {coll. }},
$$

where $m$ is the particle mass, $U$ is the externally applied field and $f(\vec{p}, \vec{x}, t)$ is the distribution function which represents the average density of particles with momentum $\vec{p}$ at the space-time point $\vec{x}, t$. The $(\delta f / \delta t)_{\text {coll. }}$ denotes the collision term. A formal solution procedure which generalizing the Chapman-Enskog method to solve Eq. (1) was given in [2] where the first and second approximations of the distribution function and expressions for the viscosity and heat conductivity coefficients were given. Here, we consider only the lowest order (first approximation) of solution of the above Boltzmann equation and requiring that the collision term in Eq. (1) to be zero, i.e., $(\delta f / \delta t)_{\text {coll. }}=0$.

The lowest order solution to Eq. (1) (with $\nabla U(\vec{x}, t)=0$ ) is given by

$$
f^{(0)}(\vec{p}, \vec{x}, t)=\left\{\exp \left[\frac{(\vec{p}-m \vec{u}(\vec{x}, t))^{2}}{2 m k_{\mathrm{B}} T(\vec{x}, t)}-\mu(\vec{x}, t) / k_{\mathrm{B}} T(\vec{x}, t)\right]+\theta\right\}^{-1},
$$

where $\theta=+1$ denotes the Fermi-Dirac statistics and $\theta=-1$ the Bose-Einstein statistics. To complete the equilibrium solution we have to determine the five unknown functions $T(\vec{x}, t), \mu(\vec{x}, t)$, and $\vec{u}(\vec{x}, t)$, which appear in Eq. (2). These five flow parameters can be determined by making use of the five conservation laws for number of particles, momentum, and energy. These five conservation laws are obtained by multiplying Eq. (1) by $1, \vec{p}$, or $\vec{p}^{2} / 2 m$, and then integrating the resulting equations over all $\vec{p}$. The integrals of the collision terms in all three cases vanish automatically and we have the differential conservation laws for the conserved macroscopic quantities, i.e., the particle number density $n(\vec{x}, t)$, the momentum density, $\vec{J}=m \vec{j}$, and the energy density, $\epsilon(\vec{x}, t)$ as follows:

$$
\begin{aligned}
& \frac{\partial n(\vec{x}, t)}{\partial t}+\nabla_{\vec{x}} \cdot \vec{j}(\vec{x}, t)=0, \\
& \frac{\partial m \vec{j}(\vec{x}, t)}{\partial t}+\nabla_{\vec{x}} \cdot \int \frac{\mathrm{d} \vec{p}}{h^{3}} \vec{p} \frac{\vec{p}}{m} f(\vec{p}, \vec{x}, t)=0, \\
& \frac{\partial \epsilon(\vec{x}, t)}{\partial t}+\nabla_{\vec{x}} \cdot \int \frac{\mathrm{d} \vec{p}}{h^{3}} \frac{\vec{p}}{m} \frac{p^{2}}{2 m} f(\vec{p}, \vec{x}, t)=0 .
\end{aligned}
$$

Here the number density, number density flux, and energy density are given, respectively, by

$$
\begin{aligned}
n(\vec{x}, t) & =\int \frac{\mathrm{d} \vec{p}}{h^{3}} f(\vec{p}, \vec{x}, t), \\
\vec{j}(\vec{x}, t) & =\int \frac{\mathrm{d} \vec{p}}{h^{3}} \frac{\vec{p}}{m} f(\vec{p}, \vec{x}, t), \\
\epsilon(\vec{x}, t) & =\int \frac{\mathrm{d} \vec{p}}{h^{3}} \frac{p^{2}}{2 m} f(\vec{p}, \vec{x}, t) .
\end{aligned}
$$


Other higher-order moments can also be defined such as stress tensor and the heat flux vector. For the local equilibrium Bose-Einstein solution, one can obtain these macroscopic quantities in closed form in terms of the Bose function, for example, see [21,22]. In this work, we shall not consider the effect of the externally applied field $U(\vec{x}, t)$. To illustrate the method, we first consider the following local equilibrium Bose-Einstein distribution in two space dimensions,

$$
f_{2}^{(0)}\left(p_{x}, p_{y}, x, y, t\right)=\left[z^{-1} \mathrm{e}^{\left[\left(p_{x}-m u_{x}\right)^{2}+\left(p_{y}-m u_{y}\right)^{2}\right] / 2 m k_{\mathrm{B}} T(x, y, t)}-1\right]^{-1},
$$

where $z(x, y, t)=\mathrm{e}^{\mu(x, y, t) / k_{\mathrm{B}} T(x, y, t)}$ is the fugacity, $u_{x}(x, y, t)$, and $u_{y}(x, y, t)$ are the mean velocity components. Then the number density $n(x, y, t)$ is given by

$$
n(x, y, t)=\int_{-\infty}^{\infty} \frac{\mathrm{d} p_{x} \mathrm{~d} p_{y}}{h^{2}} f_{2}^{(0)}\left(p_{x}, p_{y}, x, y, t\right)=\frac{g_{1}(z)}{\lambda^{2}}
$$

the momentum $\vec{j}(x, y, t)$,

$$
\begin{aligned}
& j_{x}(x, y, t)=\int_{-\infty}^{\infty} \frac{\mathrm{d} p_{x} \mathrm{~d} p_{y}}{h^{2}} \frac{p_{x}}{m} f_{2}^{(0)}\left(p_{x}, p_{y}, x, y, t\right)=n(x, y, t) u_{x}(x, y, t), \\
& j_{y}(x, y, t)=\int_{-\infty}^{\infty} \frac{\mathrm{d} p_{x} \mathrm{~d} p_{y}}{h^{2}} p_{y} m f_{2}^{(0)}\left(p_{x}, p_{y}, x, y, t\right)=n(x, y, t) u_{y}(x, y, t)
\end{aligned}
$$

and the energy density $\epsilon(x, y, t)$,

$$
\epsilon(x, y, t)=\int_{-\infty}^{\infty} \frac{\mathrm{d} p_{x} \mathrm{~d} p_{y}}{h^{2}} \frac{\left(p_{x}^{2}+p_{y}^{2}\right)}{2 m} f_{2}^{(0)}=\frac{g_{2}(z)}{\beta \lambda^{2}}+\frac{1}{2} n\left(u_{x}^{2}+u_{y}^{2}\right),
$$

where $\lambda=\sqrt{\frac{\beta h^{2}}{2 \pi m}}$ is the thermal wavelength and $\beta=1 / k_{\mathrm{B}} T(x, y, t)$.

In the above, $g_{v}(z)$ denotes the Bose function of order $v$ which is defined by

$$
g_{v}(z) \equiv \frac{1}{\Gamma(v)} \int_{0}^{\infty} \mathrm{d} x \frac{x^{v-1}}{z^{-1} \mathrm{e}^{x}-1}=\sum_{l=1}^{\infty} \frac{Z^{l}}{l^{v}} .
$$

We note that Bose functions with $v \leqslant 1$ diverge as $z \rightarrow 1$ and $g_{3 / 2}(z)$ remains finite at $z=1$ but with infinite slope while $g_{v}(z)$ with $v>3 / 2$ have finite values and slopes at $z=1$. Since we will derive beam scheme in one, two, and three space dimensions and they involve the Bose functions $g_{d / 2}(z), g_{d / 2+1}(z)$, and $g_{d / 2+2}(z)$ for the beam scheme in $d$ dimensions $(d=1,2,3)$. It is also noted that thermodynamic functions and the Bose-Einstein condensation of an ideal (interactionless) gas of $N$ bosons are peculiarly related to the space dimensionality $[23,24]$.

\section{Quantum kinetic beam scheme}

To illustrate the derivation of the kinetic beam scheme for ideal quantum gases, we first consider the formulation in two space dimensions in Cartesian coordinates. Divide the computational space into a number of cells of area $\Delta A_{i, j}$. Without loss of generality, we assume uniform rectangular cells with $\Delta x=\Delta y$ and $\Delta A_{i, j}=\Delta x \Delta y$. The local state of gas in each cell $(i, j)$ at any time $t$ is specified by $Q_{i, j}=\left(n, n u_{x}, n u_{y}, \epsilon\right)_{i, j}^{\mathrm{T}}$, which the mass density, $x$ - and $y$-momentum densities, and the energy density, respectively. We approximate the Bose-Einstein distribution in two space dimensions, $f_{2}^{(0)}$, by

$$
\begin{aligned}
f_{2}^{(0)}(\vec{p}, \vec{x}, t) \cong & q_{i, j}\left(p_{x}, p_{y}\right) \\
= & a_{i, j} \delta\left(p_{x}-p_{x 0}, p_{y}-p_{y 0}\right)+b_{i, j} \delta\left(p_{x}-p_{x 0}^{+}, p_{y}-p_{y 0}\right)+b_{i, j} \delta\left(p_{x}-p_{x 0}^{-}, p_{y}-p_{y 0}\right) \\
& +c_{i, j} \delta\left(p_{x}-p_{x 0}, p_{y}-p_{y 0}^{+}\right)+c_{i, j} \delta\left(p_{x}-p_{x 0}, p_{y}-p_{y 0}^{-}\right),
\end{aligned}
$$

where $p_{x 0}^{ \pm}=p_{x 0} \pm \Delta p_{x}$ and $p_{y 0}^{ \pm}=p_{y 0} \pm \Delta p_{y}$.

The unknown parameters $a, b, c, p_{x 0}, p_{y 0}, \Delta p_{x}$ and they are given by 


$$
\begin{aligned}
& a=\left(\frac{2 \pi m}{\beta}\right)\left[g_{1}(z)-\frac{4}{3} \frac{g_{2}^{2}(z)}{g_{3}(z)}\right], \\
& b=c=\frac{1}{3}\left(\frac{2 \pi m}{\beta}\right) \frac{g_{2}^{2}(z)}{g_{3}(z)}, \\
& \Delta p_{x}=\Delta p_{y}=\sqrt{\frac{3 m}{2 \beta} \frac{g_{3}(z)}{g_{2}(z)}} \\
& p_{x o}=m u_{x}, p_{y o}=m u_{y} .
\end{aligned}
$$

The conservative quantities carried by each beam in cell $(i, j)$ are $Q_{\sigma, i, j}=\left(R_{\sigma}, M_{\sigma}, N_{\sigma}, E_{\sigma}\right)_{i, j}$, with

$$
\begin{aligned}
R_{\sigma, i, j} & =\int \frac{\mathrm{d} p_{x} \mathrm{~d} p_{y}}{h^{2}} c_{\sigma, i, j} \delta\left(p_{x}-\bar{p}_{x, \sigma}, p_{y}-\bar{p}_{y, \sigma}\right), \\
M_{\sigma, i, j} & =\int \frac{\mathrm{d} p_{x} \mathrm{~d} p_{y}}{h^{2}} c_{\sigma, i, j} \frac{p_{x}}{m} \delta\left(p_{x}-\bar{p}_{x, \sigma}, p_{y}-p_{0 y}\right), \\
N_{\sigma, i, j} & =\int \frac{\mathrm{d} p_{x} \mathrm{~d} p_{y}}{h^{2}} c_{\sigma, i, j} \frac{p_{y}}{m} \delta\left(p_{x}-p_{0 x}, p_{y}-\bar{p}_{y, \sigma}\right), \\
E_{\sigma, i} & =\int \frac{\mathrm{d} p_{x} \mathrm{~d} p_{y}}{h^{2}} c_{\sigma, i, j} \frac{p_{x}^{2}+p_{y}^{2}}{2 m} \delta\left(p_{x}-\bar{p}_{x, \sigma}, p_{y}-\bar{p}_{y, \sigma}\right),
\end{aligned}
$$

where $\bar{p}_{x, \sigma}=p_{0 x}$, for $\sigma=1,4,5, \bar{p}_{x, 2}=p_{0 x}-\Delta p_{x}$, and $\bar{p}_{x, 3}=p_{0 x}+\Delta p_{x} ; \bar{p}_{y, \sigma}=p_{0 y}$, for $\sigma=1,2,3, \bar{p}_{y, 4}=p_{0 y}-\Delta p_{y}$, and $\bar{p}_{y, 5}=p_{0 y}+\Delta p_{y}$; and $c_{\sigma, i, j}=a_{i, j}$, for $\sigma=1$, and $c_{\sigma, i, j}=b_{i, j}$, if $\sigma=2,3,4,5$.

The beam velocities in the $x$-direction in cell $(i, j)$ are

$$
V_{1, i, j}^{x}=V_{4, i, j}^{x}=V_{5, i, j}^{x}=u_{x i, j}, \quad V_{2, i, j}^{x}=u_{x i, j}+\Delta p_{x i, j} / m, \quad V_{3, i, j}^{x}=u_{x i, j}-\Delta p_{x i, j} / m,
$$

and the beam velocities in the $y$-direction in cell $(i, j)$ are

$$
V_{1, i, j}^{y}=V_{2, i, j}^{y}=V_{3, i, j}^{y}=u_{y i, j}, \quad V_{4, i, j}^{y}=u_{y i, j}+\Delta p_{y i, j} / m, \quad V_{5, i, j}^{y}=u_{y i, j}-\Delta p_{y i, j} / m .
$$

The beam mass associated with the beam velocities are

$$
m_{1, i, j}=a_{i, j} n_{i, j} \Delta A_{i, j}, \quad m_{2, i, j}=m_{3, i, j}=m_{4, i, j}=m_{5, i, j}=b_{i, j} n_{i, j} \Delta A_{i, j} .
$$

The conservative quantities carried by each beam in cell $(i, j)$ are

$$
Q_{\sigma, i, j}=\left[\begin{array}{c}
m_{\sigma, i, j} \\
m_{\sigma, i, j} V_{\sigma, i, j}^{x} \\
m_{\sigma, i, j} V_{\sigma, i, j}^{y} \\
\frac{1}{2} m_{\sigma, i, j}\left(V_{\sigma, i, j}^{x}{ }^{2}+V_{\sigma, i, j}^{y}{ }^{2}\right)+\frac{g_{2}(z)}{\beta \lambda^{2}}
\end{array}\right]
$$

and the overall conservative quantities of gases in cell $(i, j)$ are defined as $Q_{i, j}=\sum_{\sigma=1}^{5} Q_{\sigma, i, j}$.

The kinetic beam formulation in two space dimensions in the Cartesian coordinates $(x, y)$ can be given by

$$
\partial_{t} Q_{\sigma}+\partial_{x}\left(F_{\sigma}^{+}+F_{\sigma}^{-}\right)+\partial_{y}\left(G_{\sigma}^{+}+G_{\sigma}^{-}\right)=0,
$$

where the split beam fluxes $F_{\sigma}^{ \pm}$and $G_{\sigma}^{ \pm}$at cell $(i, j)$ are defined by

$$
F_{\sigma, i, j}^{ \pm}=V_{\sigma, i, j}^{x \pm} Q_{\sigma, i, j}, \quad G_{\sigma, i, j}^{ \pm}=V_{\sigma, i, j}^{y \pm} Q_{\sigma, i, j}
$$

where $V_{\sigma}^{x \pm}=\left(V_{\sigma}^{x} \pm\left|V_{\sigma}^{x}\right|\right) / 2$ and $V_{\sigma}^{y \pm}=\left(V_{\sigma}^{y} \pm\left|V_{\sigma}^{y}\right|\right) / 2$.

The basic first-order quantum beam scheme can be expressed in the form of a conservative scheme in terms of the numerical flux

$$
Q_{\sigma, i, j}^{n+1}=Q_{\sigma, i, j}^{n}-\Delta t\left(F_{\sigma, i+1 / 2, j}^{N}-F_{\sigma, i-1 / 2, j}^{N}\right)-\Delta t\left(G_{\sigma, i, j+1 / 2}^{N}-G_{\sigma, i, j-1 / 2}^{N}\right),
$$

where the numerical fluxes are, respectively, given by 


$$
F_{\sigma, i+1 / 2, j}^{N}=F_{\sigma, i, j}^{+}+F_{\sigma, i+1, j}^{-}, \quad G_{\sigma, i, j+1 / 2}^{N}=G_{\sigma, i, j}^{+}+G_{\sigma, i, j+1}^{-} .
$$

The time step $\Delta t$ is subjected to the condition that no single beam moves farther than a cell size $\Delta A_{i, j}$ during $\Delta t$

$$
\Delta t \leqslant \min \left(\frac{\Delta x}{\left|V_{\sigma}^{x}\right|}, \frac{\Delta y}{\left|V_{\sigma}^{y}\right|}\right)
$$

The final 2D governing equations in generalized coordinates $(\xi, \eta)$ based on the beam splitting method can be expressed as:

$$
\partial_{t} \hat{Q}_{\sigma}+\partial_{\xi}\left(\hat{F}_{\sigma}^{+}+\hat{F}_{\sigma}^{-}\right)+\partial_{\eta}\left(\hat{G}_{\sigma}^{+}+\hat{G}_{\sigma}^{-}\right)=0
$$

where $\hat{Q}_{\sigma}=Q_{\sigma} / J, \hat{F}_{\sigma}^{ \pm}=\left(\xi_{x} F_{\sigma}^{ \pm}+\xi_{y} G_{\sigma}^{ \pm}\right) / J, \hat{G}_{\sigma}^{ \pm}=\left(\eta_{x} F_{\sigma}^{ \pm}+\eta_{y} G_{\sigma}^{ \pm}\right) / J$, and $J=\xi_{x} \eta_{y}-\xi_{y} \eta_{x}$ is the metric Jacobian.

The beam velocities in the generalized coordinates at grid point $(i, j)$ are

$$
V_{\sigma}^{\xi}=\xi_{x} V_{\sigma}^{x}+\xi_{y} V_{\sigma}^{y}, \quad V_{\sigma}^{\eta}=\eta_{x} V_{\sigma}^{x}+\eta_{y} V_{\sigma}^{y} .
$$

Define $V_{\sigma}^{\xi \pm}=\left(V_{\sigma}^{\xi} \pm\left|V_{\sigma}^{\xi}\right|\right) / 2$ and $V_{\sigma}^{\eta \pm}=\left(V_{\sigma}^{\eta} \pm\left|V_{\sigma}^{\eta}\right|\right) / 2$. Then we have $\hat{F}_{\sigma}^{ \pm}=V_{\sigma}^{\xi \pm} \hat{Q}_{\sigma}$ and $\hat{G}_{\sigma}^{ \pm}=V_{\sigma}^{\eta \pm} \hat{Q}_{\sigma}$.

Without causing any ambiguity, we can omit the hat signs below. The first-order quantum beam scheme can be expressed in the form of a conservative scheme in terms of the numerical flux

$$
Q_{\sigma, i, j}^{n+1}=Q_{\sigma, i, j}^{n}-\Delta t\left(F_{\sigma, i+1 / 2, j}^{N}-F_{\sigma, i-1 / 2, j}^{N}\right)-\Delta t\left(G_{\sigma, i, j+1 / 2}^{N}-G_{\sigma, i, j-1 / 2}^{N}\right),
$$

where the numerical fluxes are respectively given by

$$
F_{\sigma, i+1 / 2, j}^{N}=F_{\sigma, i, j}^{+}+F_{\sigma, i+1, j}^{-}, \quad G_{\sigma, i, j+1 / 2}^{N}=F_{\sigma, i, j}^{+}+F_{\sigma, i, j+1}^{-} .
$$

The time step is determined by

$$
\Delta t \leqslant \min \left(\frac{1}{\max \left|V_{\sigma, i, j}^{\xi}\right|}, \frac{1}{\max \left|V_{\sigma, i, j}^{\eta}\right|}\right) .
$$

For explicit methods in multiple space dimensions, the Strang-type dimensional splitting was employed and the integrating scheme can be expressed in terms of operators as

$$
Q_{\sigma, i, j}^{n+2}=L_{\xi}(\Delta t) L_{\eta}(\Delta t) L_{\eta}(\Delta t) L_{\xi}(\Delta t) Q_{\sigma, i, j}^{n} .
$$

The one-dimensional operator in the $\xi$-direction $L_{\xi}$ is defined by

$$
L_{\xi}(\Delta t) Q_{\sigma, i, j}^{n}=Q_{\sigma, i, j}^{n}-\Delta t\left(F_{\sigma, i+1 / 2, j}^{N}-F_{\sigma, i-1 / 2, j}^{N}\right) .
$$

Similar expressions can be given for the $L_{\eta}$ operator and the numerical flux in the $\eta$-direction.

Formulations in three space dimensions in general coordinates $(\xi, \eta, \zeta)$ system are given in Appendix A.

In the case of Fermi-Dirac distribution, we simply replace the Bose function above by the following Fermi function:

$$
f_{v}(z) \equiv \frac{1}{\Gamma(v)} \int_{0}^{\infty} \mathrm{d} x \frac{x^{v-1}}{z^{-1} \mathrm{e}^{x}+1} \sum_{l=1}^{\infty}-\frac{(-Z)^{l}}{l^{v}} .
$$

Following the same procedure,we find that we can just replace Bose function with Fermi function to simulate the Fermi-Dirac gas. In Bose-Einstein gas, the fugacity could not greater than one or less than zero, but in Fermi-Dirac gas we do not have such limits.

The calculation for fugacity $z$ can be done by combination of Eqs. (10)-(13) in dimensionless form as [20]. Substitute Eqs. (10)-(12) into Eq. (13), We can get the following equation:

$$
\chi_{2}=\epsilon-\frac{j_{x}^{2}+j_{y}^{2}}{2 n}-\left(\frac{n}{g_{1}(z)}\right)^{2} g_{2}(z)=0 .
$$

The fugacity is obtained by solving this equation numerically. 


\section{Implementation of high resolution schemes}

The above scheme is of first-order accuracy and for practical applications we need high-order methods. In this section, we adopt the total variation diminishing [25] method and essentially non-oscillatory interpolation method developed by Harten et al. [26] to the basic first-order quantum beam scheme to result in a class of high resolution methods for the computation of quantum ideal gas dynamical flows.

Following [17,31], we consider the numerical flux in $\xi$ direction of a class of high resolution schemes based on essentially non-oscillatory interpolation concept as follows and for simplicity we omit the $j$ subindexes.

$$
F_{\sigma, i+1 / 2}^{N}=\frac{1}{2}\left(F_{\sigma, i}^{n}+F_{\sigma, i+1}^{n}+\phi_{\sigma, i+1 / 2}\right),
$$

where

$$
\phi_{\sigma, i+\frac{1}{2}}=\sigma\left(\bar{a}_{\sigma, i+\frac{1}{2}}\right)\left(g_{\sigma, i}+g_{\sigma, i+1}\right)+\omega \bar{\sigma}\left(\bar{a}_{\sigma, i+\frac{1}{2}}\right)\left(d_{\sigma, i}+d_{\sigma, i+1}\right)-\psi\left(\bar{a}_{\sigma, i+\frac{1}{2}}+\gamma_{\sigma, i+\frac{1}{2}}+\omega \delta_{\sigma, i+\frac{1}{2}}\right) \Delta_{+} Q_{\sigma, i}^{n} .
$$

The formulations of the limiter functions, $g_{\sigma, i}$ and $d_{\sigma, i}$, and characteristic speed functions, $\sigma(\bar{a}), \bar{\sigma}(\bar{a}), \gamma(\bar{a})$, $\delta(\bar{a})$, and functions $m(x, y), \bar{m}(x, y)$ may refer the reference [17], or to be described as follows.

The limiter functions $g_{\sigma, i}$ and $d_{\sigma, i}$ are defined as

$$
\begin{aligned}
g_{\sigma, i} & =m\left(\Delta_{+} Q_{\sigma, i}^{n}-\vartheta \bar{m}\left(\Delta_{-} \Delta_{+} Q_{\sigma, i}^{n}, \Delta_{+} \Delta_{+} Q_{\sigma, i}^{n}\right), \Delta_{-} Q_{\sigma, i}^{n}+\vartheta \bar{m}\left(\Delta_{-} \Delta_{-} Q_{\sigma, i}^{n}, \Delta_{+} \Delta_{-} Q_{\sigma, i}^{n}\right)\right), \\
d_{\sigma, i} & = \begin{cases}\bar{m}\left(\Delta_{-} \Delta_{-} Q_{\sigma, i}^{n}, \Delta_{+} \Delta_{-} Q_{\sigma, i}^{n}\right) & \text { if }\left|\Delta_{-} Q_{\sigma, i}^{n}\right| \leqslant\left|\Delta_{+} Q_{\sigma, i}^{n}\right|, \\
\bar{m}\left(\Delta_{-} \Delta_{+} Q_{\sigma, i}^{n}, \Delta_{+} \Delta_{+} Q_{\sigma, i}^{n}\right) & \text { if }\left|\Delta_{-} Q_{\sigma, i}^{n}\right|>\left|\Delta_{+} Q_{\sigma, i}^{n}\right| .\end{cases}
\end{aligned}
$$

The functions $m(x, y), \bar{m}(x, y)$ in above equations are defined as

$$
\begin{aligned}
& m(a, b)= \begin{cases}s \min (|a|,|b|) & \text { if } \operatorname{sign}(a)=\operatorname{sign}(b)=s, \\
0 & \text { otherwise, }\end{cases} \\
& \bar{m}(a, b)= \begin{cases}a & \text { if }|a| \leqslant|b|, \\
b & \text { if }|a|>|b|,\end{cases}
\end{aligned}
$$

where $a$ and $b$ are arbitrary real numbers. The other variables and functions in Eq. (42) are defined as follows:

$$
\begin{aligned}
& \bar{a}_{\sigma, i+\frac{1}{2}}=\bar{a}\left(Q_{\sigma, i}^{n}, Q_{\sigma, i+1}^{n}\right)= \begin{cases}F_{\sigma, i+1}^{n}-F_{\sigma, i}^{n} & \text { if } Q_{\sigma, i}^{n} \neq Q_{\sigma, i+1}^{n}, \\
Q_{\sigma, i}^{n}-Q_{\sigma, i}^{n} & \text { if } Q_{\sigma, i}^{n}=Q_{\sigma, i+1}^{n} .\end{cases} \\
& \psi(z)= \begin{cases}|z| & \text { if }|z| \geqslant \epsilon, \\
\left(z^{2}+\epsilon^{2}\right) / 2 \epsilon & \text { if }|z|<\epsilon,\end{cases} \\
& \sigma(z)=\left(\psi(z)-\lambda z^{2}\right) / 2, \\
& \bar{\sigma}(z)= \begin{cases}\left(\lambda^{2}|z|^{3}-3 \lambda|z|^{2}+2|z|\right) / 6 & \text { if }\left|\Delta_{-} v_{i}^{n}\right| \leqslant\left|\Delta_{+} v_{i}^{n}\right|, \\
\left(\lambda^{2}|z|^{3}-|z|\right) / 6 & \text { if }\left|\Delta_{-} v_{i}^{n}\right|>\left|\Delta_{+} v_{i}^{n}\right|,\end{cases} \\
& \gamma_{\sigma, i+\frac{1}{2}}= \begin{cases}\sigma\left(\bar{a}_{\sigma, i+\frac{1}{2}}\right)\left(g_{\sigma, i+1}-g_{\sigma, i}\right) / \Delta_{+} Q_{\sigma, i}^{n} & \text { if } \Delta_{+} Q_{\sigma, i}^{n} \neq 0, \\
0 & \text { otherwise, }\end{cases} \\
& \delta_{\sigma, i+\frac{1}{2}}= \begin{cases}\bar{\sigma}\left(\bar{a}_{\sigma, i+\frac{1}{2}}\right)\left(d_{\sigma, i+1}-d_{\sigma, i}\right) / \Delta_{+} Q_{\sigma, i}^{n} & \text { if } \Delta_{+} Q_{\sigma, i}^{n} \neq 0, \\
0 & \text { otherwise, }\end{cases} \\
& \Delta_{ \pm} Q_{\sigma, i}^{n}= \pm\left(Q_{\sigma, i \pm 1}^{n}-Q_{\sigma, i}^{n}\right),
\end{aligned}
$$

where $\epsilon$ is a small positive real number. The class of high resolution schemes covered by Eq. (42) includes the total variation diminishing (TVD) and essentially non-oscillatory (ENO) schemes, which may be selected by setting the parameters $\omega$ and $\vartheta$. 


$$
\begin{array}{ll}
\omega=0, & \vartheta=0 \rightarrow \mathrm{TVD} 2 \\
\omega=0, & \vartheta=0.5 \rightarrow \mathrm{ENO} 2, \\
\omega=1, & \vartheta=0 \rightarrow \mathrm{ENO} 3 .
\end{array}
$$

For $\omega=0$ and $\vartheta=0$, one has a second-order TVD scheme; for $\omega=0$ and $\vartheta=\frac{1}{2}$, one has a second-order ENO scheme; and for $\omega=1$ and $\vartheta=0$, one has a third-order ENO scheme.

Another type of high resolution scheme based on efficient weighted ENO methods $[27,28]$ can also be constructed. Here we consider the third-order $(r=2)$ and the fifth-order $(r=3)$ WENO methods as follows. The advantage of using WENO interpolation is that we can directly working on the split flux vectors $F^{ \pm}$and the formulation is extremely simple. The implementation of WENO methods has been presented in [20] for the case of one space dimension. Here we will not repeat the formulations.

\section{Boundary and initial conditions}

In this section, we describe the characteristic boundary conditions at the solid surface and at the far field and the initial set up of the problem. To be more specific, we consider the shock wave reflection by a circular cylinder in which the cylinder wall surface is at $j=1$ and the outer far field boundary is $j=J$. Due to the finite computational domain used, a non-reflecting boundary condition at far field needs to be employed. The upwind feature of the basic beam scheme provides a natural way to implement the non-reflecting boundary condition. At the outer far field boundary, we have

$$
Q_{i, J}^{*}=Q_{i, J}^{n}-\Delta t\left(G_{i, J}^{+}-G_{i, J-1}^{+}\right) .
$$

At the cylinder surface, the integrating scheme defined by Eq. (32) gives

$$
Q_{i, 1}^{*}=Q_{i, 1}^{n}-\Delta t\left(G_{i, 2}^{-}-G_{i, 1}^{-}\right),
$$

where $G_{i, j}^{ \pm}=\sum_{\sigma=1}^{5} G_{\sigma, i, j}^{ \pm}$.

This only partially update the state vector $Q$ since information carried by positive eigenvalues are not counted and additional conditions are needed to supplement in order to completely update the state variable $Q$ at the new time level.

In this work, we employed the equation of state

$$
P V_{d}=g U_{d}, \quad g=2 / d,
$$

where $P$ is the gas pressure, $V_{d}$ the system volume, $d$ is the space dimensionality, and $U_{d}$ is the internal energy, and here we have $d=2$, thus $g=1$. We express the boundary and initial condition in terms of parameter $g$ instead of specific heat in classical gas [30]. The boundary conditions on the surface of cylinder are as following

$$
\begin{aligned}
& V_{\eta}^{n+1}-\frac{2 c^{n+1}}{g}=V_{\eta}^{*}-\frac{2 c^{*}}{g}, \\
& \frac{p^{n+1}}{\left(\rho^{n+1}\right)^{g+1}}=\frac{p^{*}}{\left(\rho^{*}\right)^{g+1}}, \\
& U_{\eta}^{n+1}=U_{\eta}^{*}
\end{aligned}
$$

and the surface tangency condition

$$
V_{\eta}^{n+1}=0
$$

where $V_{\eta}=V /|\nabla \eta|$ and $U_{\eta}=\left(\eta_{y} u-\eta_{x} v\right) /|\nabla \eta|$.

The initial position of the incident shock wave is arbitrarily located at certain distance to the left of the cylinder. The conditions ahead of (state 1) and behind (state 2) a moving shock are related by 


$$
\begin{aligned}
& \frac{p_{2}}{p_{1}}=\frac{2(g+1) M_{s}^{2}-g}{g+2}, \\
& \frac{\rho_{2}}{\rho_{1}}=\frac{G\left(p_{2} / p_{1}\right)+1}{G+\left(p_{2} / p_{1}\right)}, \\
& u_{2}=M_{s}\left[1-\frac{g M_{s}^{2}+2}{(g+2) M_{s}^{2}}\right] c_{1},
\end{aligned}
$$

where $G=(g+2) / g$ and $c_{1}=\left((g+1) p_{1} / \rho_{1}\right)^{1 / 2}$.

Initially, when $t=0$, the fugacity at state $1\left(z_{1}\right)$ is assigned, then $n_{1}, p_{1}, T_{1}$ and $\epsilon_{1}$ can be calculated by the following equations which are derived from state equation with an assigned value of $M_{\mathrm{s}}$ and a given value of $g$ for a particular quantum gas.

$$
\begin{aligned}
T_{1} & =\frac{g_{1}(z)}{g_{2}(z)(g+1) g}, \quad n_{1}=T_{1} g_{1}(z), \quad p_{1}=\frac{n_{1}}{g+1}, \\
\epsilon_{1} & =T_{1}^{2} g_{2}(z)+\frac{1}{2} n_{1}\left(u_{1}^{2}+v_{1}^{2}\right) .
\end{aligned}
$$

The velocity components of state 1 are set to zero, $u_{1}=v_{1}=0$. The conditions at state 2 are calculated by Eq. (63) and the energy density $\epsilon_{2}$, temperature $T_{2}$ and fugacity $z$ are given as previous procedure.

Moving shock relations are applied to both sides of the incident shock and the consequent movement of motion is simulated without imposing any explicit equation of motion for the incident shock.

\section{Numerical examples and discussions}

In this section, we report some numerical examples to illustrate the performance of the present high resolution quantum beam schemes in general coordinates. For validation and comparison purposes, we first apply the numerical methods to quantum shock tube flows in one space dimension. This has been done in [20] and will not be repeated here. After validation, we apply the method to simulate a two-dimensional complex unsteady shock wave diffraction by a circular cylinder placed in a quantum gas.

Example 1 (shock wave diffraction in ideal Bose-Einstein gas). In this problem we consider a plane shock wave located initially at a certain distance ahead of the circular cylinder that propagates with shock Mach number $M_{\mathrm{s}}=2.0$ toward the cylinder and experiences transient shock diffraction. For the classical gas, an experimental study has been given in [29] and a detailed numerical simulation has been reported in [30]. A simple cylindrical grid system of $361 \times 241$ was used, consisting of 361 rays around the cylinder and 241 circles between the cylinder surface and outer boundary which is slightly stretched with $\Delta r_{\min }=0.007$. The diameter of the cylinder is 1.0 and the distance between the origin of the cylinder and the outer boundary is 7.0.

First, we report the results for the case of Bose-Einstein gas. The initial conditions are set as $M_{\mathrm{s}}=2.0$ and fugacity $z_{1}=0.8$. The flow quantities of state 1 and 2 are as follows:

$$
\begin{aligned}
& \left(\rho_{1}, u_{1}, \epsilon_{1}, T_{1}\right)=(1.205,0.000,0.603,0.749), \\
& \left(\rho_{2}, u_{2}, \epsilon_{2}, T_{2}\right)=(2.410,1.000,4.214,1.763) .
\end{aligned}
$$

The fugacity in state 2 is 0.744 . In Fig. 1 , the number density isolines obtained by the ENO2 scheme at a series of time are shown. In Fig. 1a, the incident shock is about to hit the cylinder surface and the number density contours is constant on either side of the moving shock. Figs. 1b-h show the subsequent development of the diffraction process that covers regular reflection, transition to Mach reflection, the Mach shocks collision at the wake, and the complex shock on shock interaction. The primary incident shock (I.S.), reflected bow shock (R.S.), Mach shock (M.S.), contact discontinuity (C.D.), and vortex (V.) can be easily identified. Fig. 2 shows the detail view of density contours (upper) and the gray scale map of gradient of density (lower) in the downstream of the cylinder wake at time $t=1.7$. It is observed that the complicated flow interaction resulting in Mach shocks, second contact discontinuities, and triple point were well captured. 
a

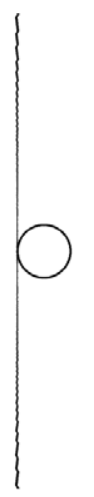

b

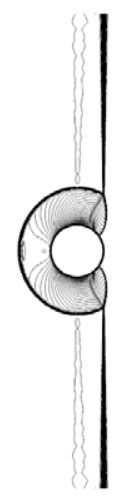

C

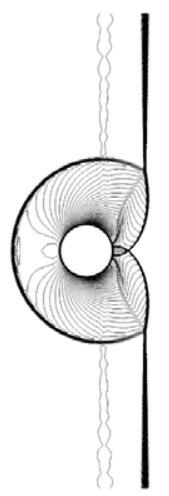

e

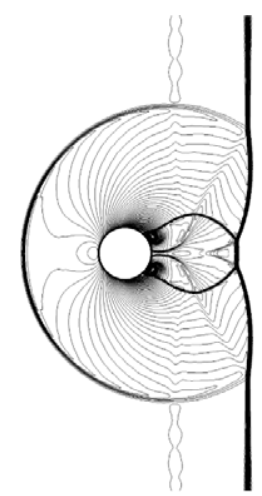

f

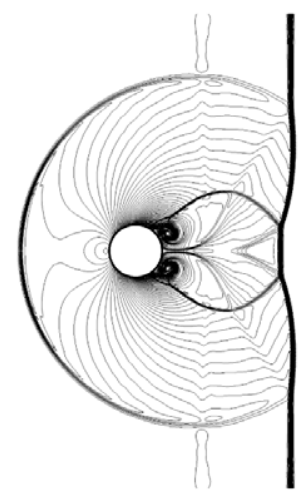

g

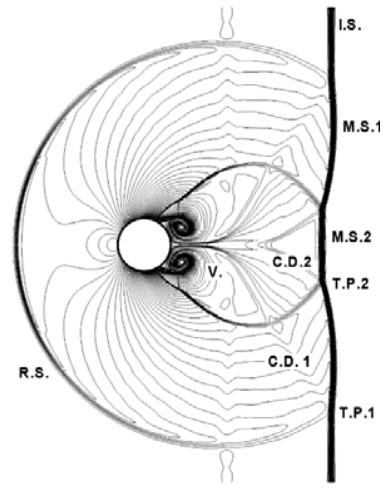

d

h

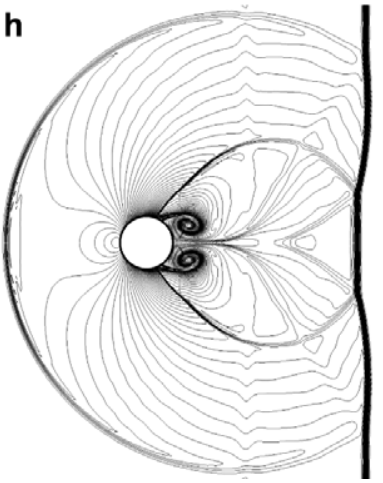

Fig. 1. Number density isolines of shock diffraction on a cylinder with $\mathrm{M}_{s}=2.0$ and fugacity $z_{1}=0.8$ for Bose-Einstein gas. (I.S. $=$ incident shock, R.S. $=$ reflected shock, M.S. $=$ Mach shock, C.D. $=$ contact discontinuity, T.P. $=$ triple point, V. $=$ vortex) 


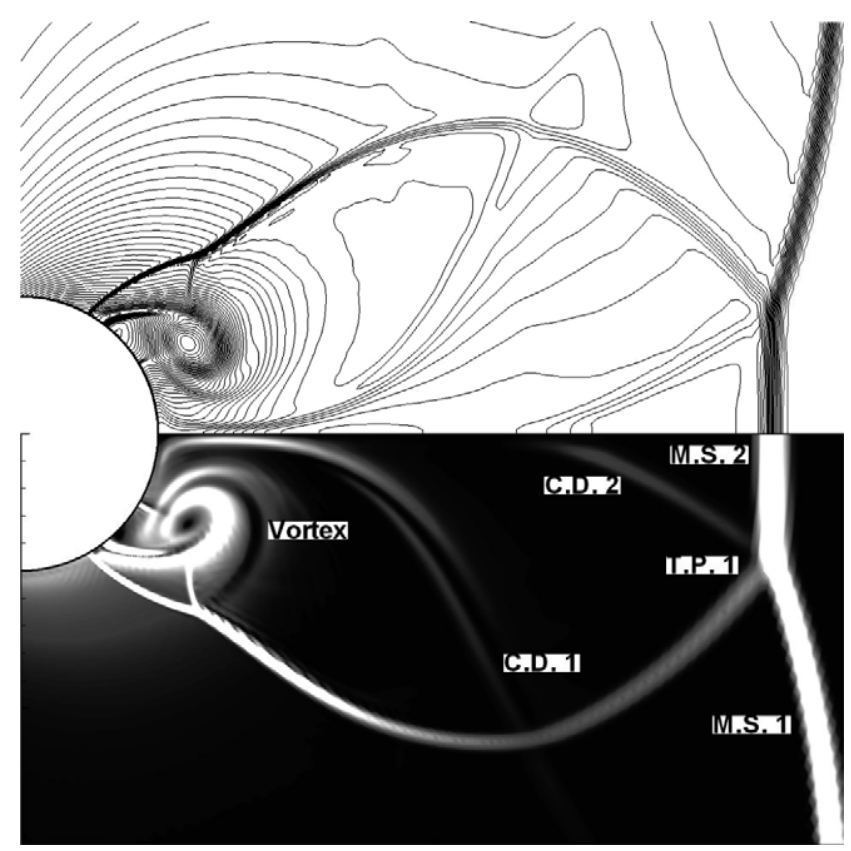

Fig. 2. Detail view of density contours (upper) and the gray scale map of gradient of density (lower) in the downstream of the cylinder wake at time $t=1.7$.
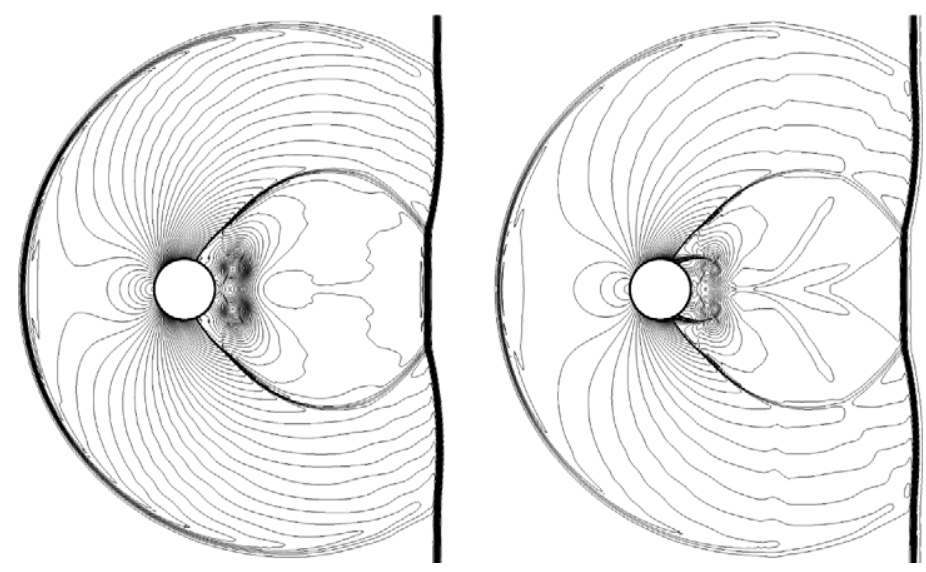

Fig. 3. Pressure (left) and temperature (right) isolines of shock diffraction on a cylinder with $M_{\mathrm{s}}=2.0$ and fugacity $z_{1}=0.8$ at time 2.3 .

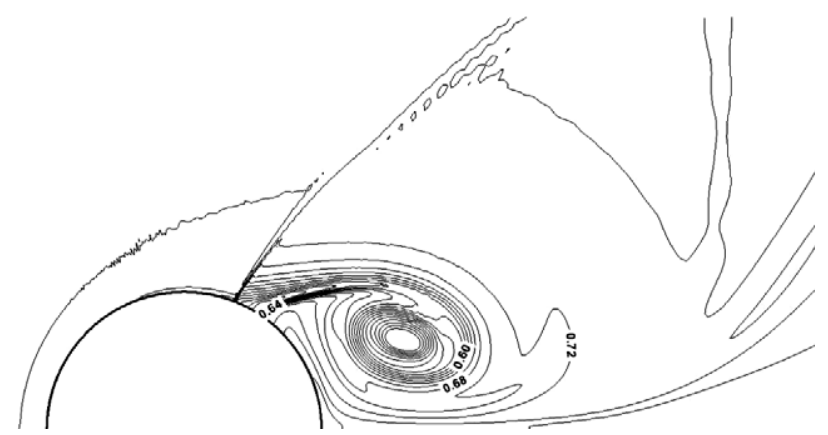

Fig. 4. Detail view of fugacity contours in the downstream of the cylinder wake, Bose-Einstein gas with $z_{1}=0.8$. 


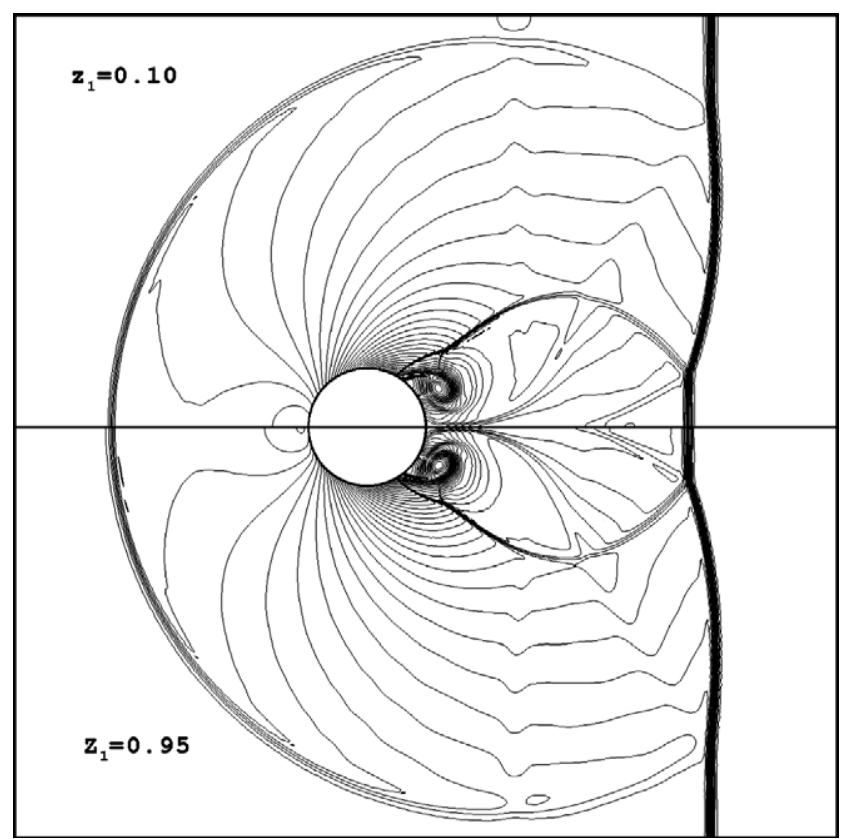

Fig. 5. Comparison with flow patterns of the two cases with fugacity $z_{1}=0.1$ and 0.95 , number density isolines of shock diffraction on a cylinder with $M_{\mathrm{s}}=2.0$ at time 1.7

Additional contour plots such as pressure and temperature are shown in Fig. 3 to assist the analysis of flow field. Various contours are shown to reveal the complex phenomenon. Fig. 4 shows the detail view of fugacity contours in the cylinder wake. It can be seen that the fugacity does not obviously change except in the vortex zone, where the value is decreasing toward the vortex center. The fugacity at vortex center is under 0.4.

We also report results for the cases of Bose-Einstein gas with two different fugacity $z_{1}=0.1$ and 0.95 . The shock Mach number is 2.0, too. The initial flow quantities of states 1 and 2 of case $z_{1}=0.1$ are as follows:

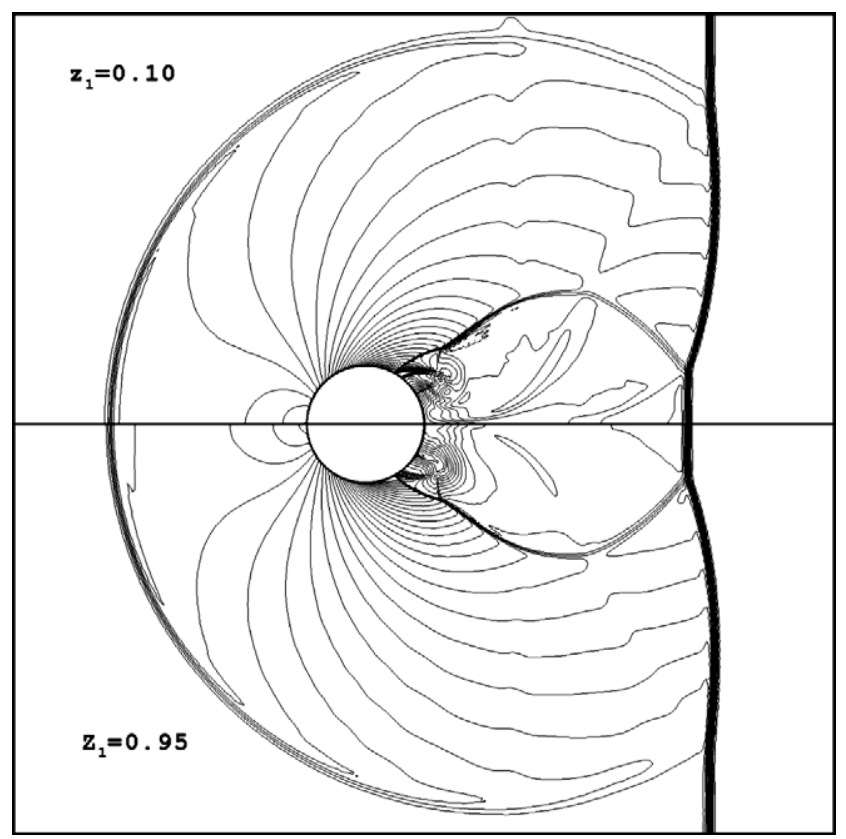

Fig. 6. Comparison with flow patterns of the two cases with fugacity $z_{1}=0.1$ and 0.95 , temperature isolines of shock diffraction on a cylinder with $M_{\mathrm{s}}=2.0$ at time 1.7 . 
a

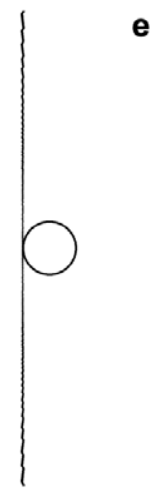

b

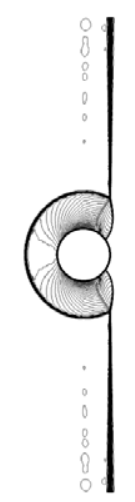

C

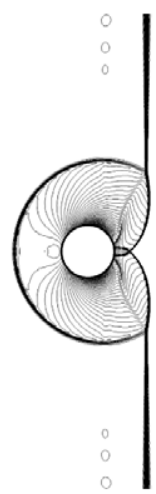

d
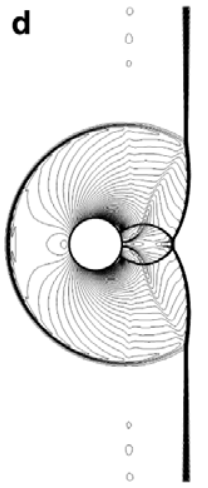

$\mathbf{f}$

g

h
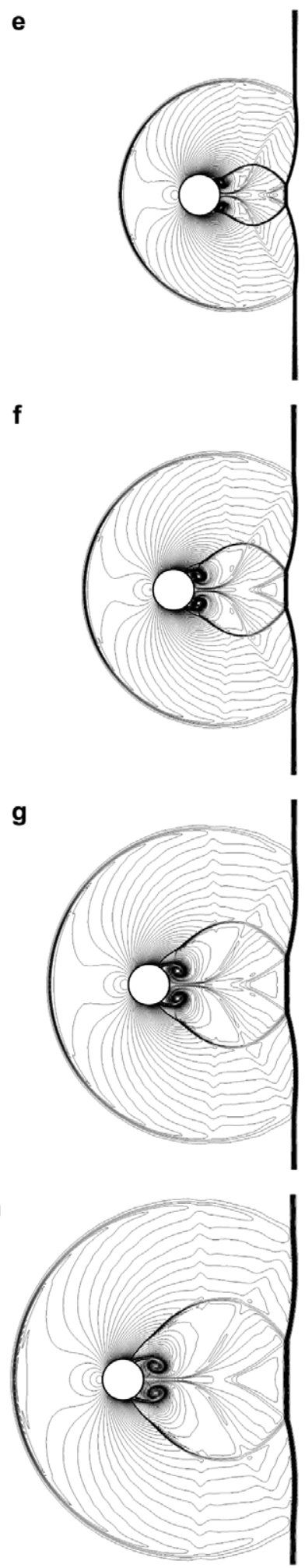

Fig. 7. Number density isolines of shock diffraction on a cylinder with $M_{\mathrm{s}}=2.0$ and fugacity $z_{1}=0.1$ for Fermi-Dirac gas. 


$$
\begin{aligned}
& \left(\rho_{1}, u_{1}, \epsilon_{1}, T_{1}\right)=(0.054,0.000,0.027,0.513), \\
& \left(\rho_{2}, u_{2}, \epsilon_{2}, T_{2}\right)=(0.108,1.000,0.189,1.277),
\end{aligned}
$$

and that for the case $z_{1}=0.95$ are as follows:

$$
\begin{aligned}
& \left(\rho_{1}, u_{1}, \epsilon_{1}, T_{1}\right)=(3.114,0.000,1.557,1.040), \\
& \left(\rho_{2}, u_{2}, \epsilon_{2}, T_{2}\right)=(6.229,1.000,7.787,2.383) .
\end{aligned}
$$

The isoline of number density and temperature of the two cases with fugacity $z_{1}=0.1$ and 0.95 are shown in Figs. 5 and 6. The minimum and maximum level of number density contours ranged from 0.02 to 0.18 for the case of $z_{1}=0.1$, and from 1.2 to 9.4 for the case of $z_{1}=0.95$. The number of levels is 41 for both cases. The flow patterns are very similar. It can be seen from the figures that the computing results of the flow structure are very similar in the classical and nearly degenerate limit.

Example 2 (shock wave diffraction in ideal Fermi-Dirac gas). Next, we report corresponding results for the case of Fermi-Dirac gas. The initial conditions are set as $M_{\mathrm{s}}=2.0$ and fugacity $z_{1}=0.1$. The flow quantities of states 1 and 2 are as follows:

$$
\begin{aligned}
& \left(\rho_{1}, u_{1}, \epsilon_{1}, T_{1}\right)=(0.047,0.000,0.023,0.488), \\
& \left(\rho_{2}, u_{2}, \epsilon_{2}, T_{2}\right)=(0.093,1.000,0.163,1.226) .
\end{aligned}
$$
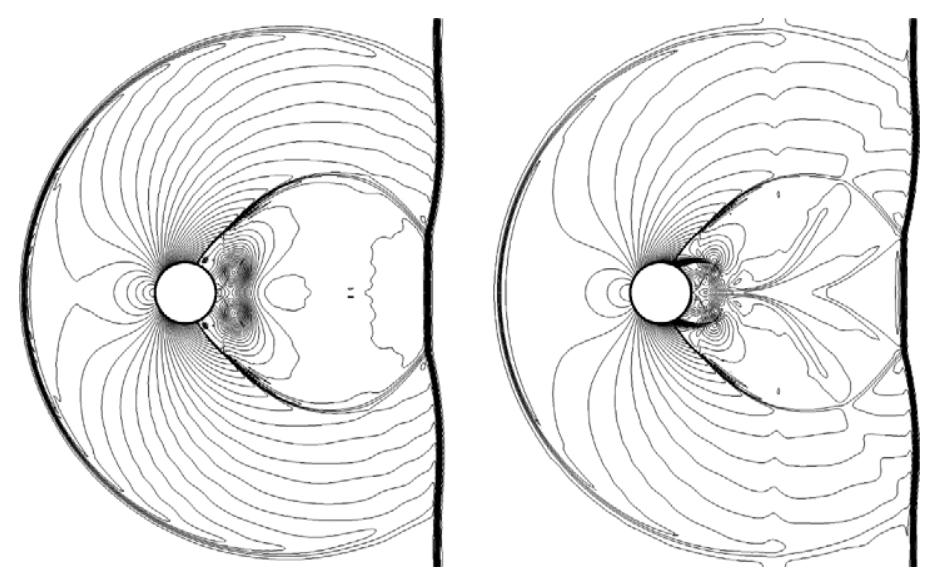

Fig. 8. Fermi-Dirac gas: Pressure (left) and temperature (right) isolines of shock diffraction on a cylinder with $M_{\mathrm{s}}=2.0$ and fugacity $z_{1}=0.1$ at time 2.3 .

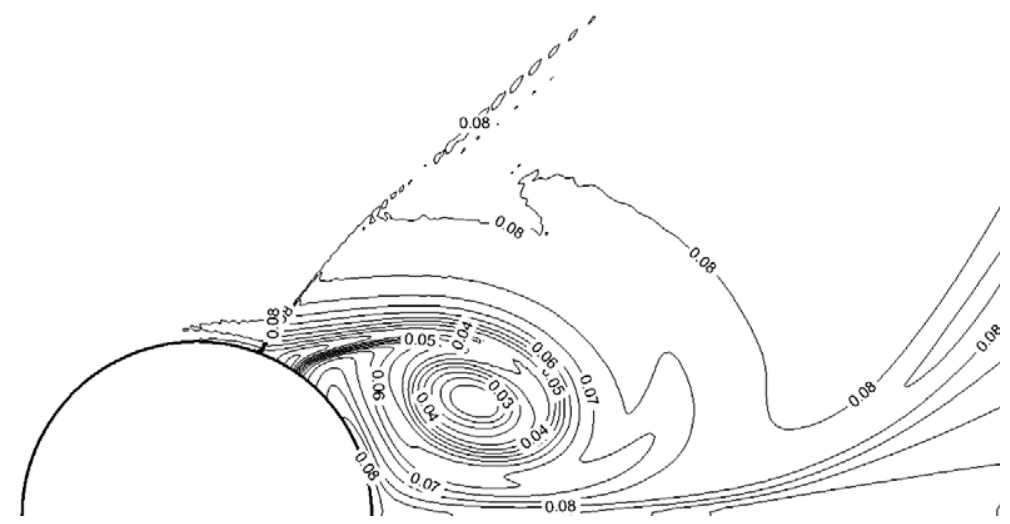

Fig. 9. Detail view of fugacity contours in the downstream of the cylinder wake, Fermi-Dirac gas with $z_{1}=0.1$. 

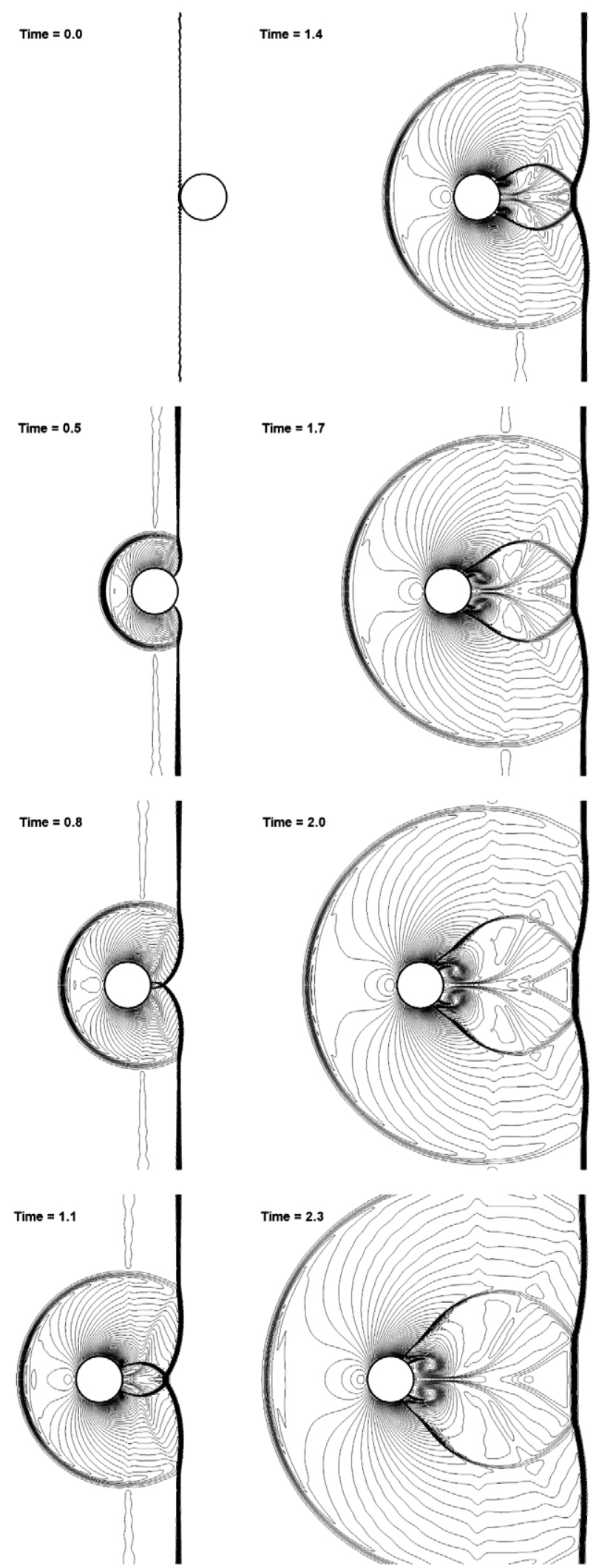

Fig. 10. Number density isolines of shock diffraction on a cylinder with $M_{\mathrm{S}}=2.0$ and fugacity $z_{1}=0.8$ for Fermi-Dirac gas. 
The fugacity in state 2 is 0.079 . In Fig. 7, the number density isolines obtained by the ENO2 scheme at a series of times are shown. The development of the diffraction process similar to the case of Bose-Einstein gas that covers regular reflection, transition to Mach reflection, the Mach shocks collision at the wake, and the complex shock on shock interaction. Additional contour plots such as pressure and temperature are shown in Fig. 8 to assist the analysis of flow field. Various contours are shown to reveal the complex phenomenon. Fig. 9 shows the detail view of fugacity contours in the cylinder wake. It can be seen that the fugacity does not change obviously except in the vortex zone, where the value is decreasing toward the vortex center where it is under 0.02 . The last case considered is that the fugacity $z_{1}=0.8$ and the flow conditions of states 1 and 2 are as follows:

$$
\begin{aligned}
& \left(\rho_{1}, u_{1}, \epsilon_{1}, T_{1}\right)=(0.254,0.000,0.127,0.432), \\
& \left(\rho_{2}, u_{2}, \epsilon_{2}, T_{2}\right)=(0.508,1.000,0.889,1.116) .
\end{aligned}
$$

The fugacity in state 2 is 0.576 . In Fig. 10, the number density isolines obtained by the ENO2 scheme at a series of times are shown for the case $z_{1}=0.8$. The development of the diffraction process similar to the case of $z_{1}=0.1$ that covers regular reflection, transition to Mach reflection, the Mach shocks collision at the wake, and the complex shock on shock interaction. The pressure and temperature contour plots are shown in Fig. 11 to assist the analysis of flow field. Fig. 12 shows the detail view of fugacity contours in the cylinder wake. At the vortex center, the fugacity is under 0.28 .
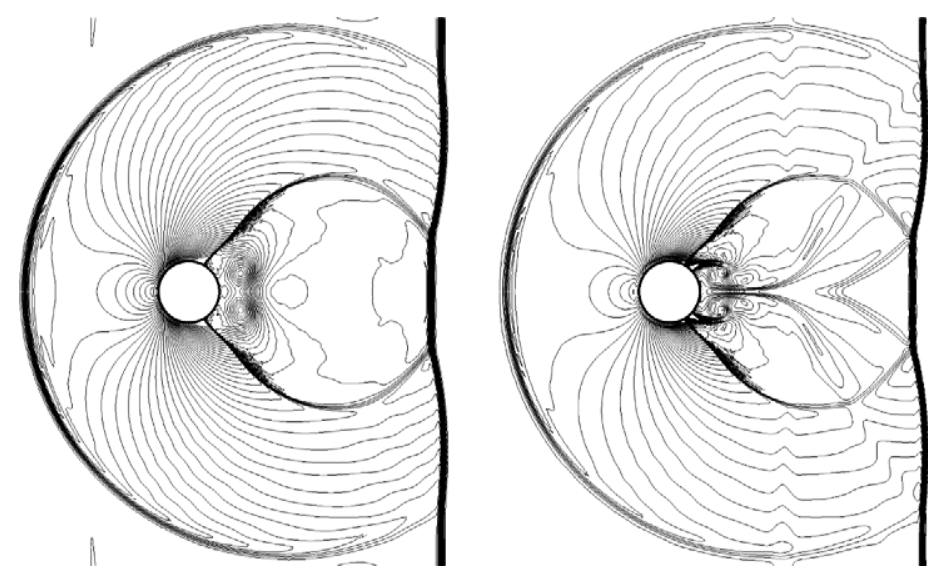

Fig. 11. Fermi-Dirac gas: Pressure (left) and temperature (right) isolines of shock diffraction on a cylinder with $M_{\mathrm{s}}=2.0$ and fugacity $z_{1}=0.8$ at time 2.3 .

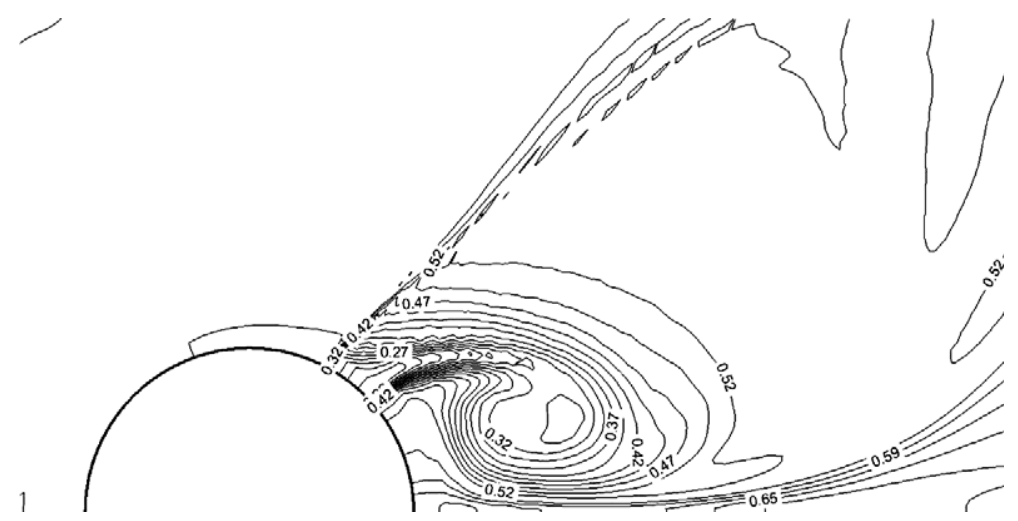

Fig. 12. Detail view of fugacity contours in the downstream of the cylinder wake, Fermi-Dirac gas with $z_{1}=0.8$. 
In summary, the complete shock wave diffraction patterns generated by a moving shock wave impinging upon a circular cylinder in ideal quantum gases, which exhibit shock wave reflection, contact surface and expansion wave and their complex interaction, can be adequately resolved and captured by the present high resolution quantum beam schemes.

\section{Concluding remarks}

In this work, a class of high resolution kinetic beam schemes in generalized coordinates system have been devised for the computation of practical quantum gas dynamical flows. The kinetic beam scheme for ideal quantum gas was first cast in the form of a flux splitting method and then general coordinate transformation was introduced as those done in classical gas dynamics or inviscid aerodynamics. The essentially non-oscillatory interpolation concept was adopted for the spatial flux difference to yield a class of high resolution schemes. The resulting method was applied to simulate unsteady shock wave diffraction by a circular cylinder to investigate the complex nonlinear manifestation of shock wave, contact surface, and expansion wave and their interactions. The complete diffraction process was followed after the incident moving shock has travelled several times of the cylinder diameter and the flow patterns were depicted through a series of density contours. Both Bose-Einstein and Fermi-Dirac gases were considered. The simulated results indicate that the present high resolution quantum beam schemes can resolve the flow structures accurately thus they may provide a valuable tool for exploring various ideal quantum gas dynamical flow problems, particularly, when there are very few experimental data available. Formulations for general coordinates in three space dimensions are also included in Appendix A.

\section{Acknowledgment}

This work is done under the auspices of National Science Council, Taiwan through Grant NSC 93-2212E002-040.

\section{Appendix A}

We approximate the Bose-Einstein distribution in three space dimensions, $f_{3}^{(0)}$, in cell $(i, j, k)$ by

$$
\begin{aligned}
f_{3}^{(0)}(\vec{p}, \vec{x}, t) \cong & q_{i, j, k}\left(p_{x}, p_{y}, p_{z}\right)=a_{i, j, k} \delta\left(p_{x}-p_{x 0}, p_{y}-p_{y 0}, p_{z}-p_{z 0}\right)+b_{i, j, k} \delta\left(p_{x}-p_{x 0}^{+}, p_{y}-p_{y 0}, p_{z}-p_{z 0}\right) \\
& +b_{i, j, k} \delta\left(p_{x}-p_{x 0}^{-}, p_{y}-p_{y 0}, p_{z}-p_{z 0}\right)+c_{i, j, k} \delta\left(p_{x}-p_{x 0}, p_{y}-p_{y 0}^{+}, p_{z}-p_{z 0}\right) \\
& +c_{i, j, k} \delta\left(p_{x}-p_{x 0}, p_{y}-p_{y 0}^{-}, p_{z}-p_{z 0}\right)+d_{i, j, k} \delta\left(p_{x}-p_{x 0}, p_{y}-p_{y 0}, p_{z}-p_{z 0}^{+}\right) \\
& +d_{i, j, k} \delta\left(p_{x}-p_{x 0}, p_{y}-p_{y 0}, p_{z}-p_{z 0}^{-}\right)
\end{aligned}
$$

where $p_{x 0}^{ \pm}=p_{x 0} \pm \Delta p_{x}, p_{y 0}^{ \pm}=p_{y 0} \pm \Delta p_{y}$, and $p_{z 0}^{ \pm}=p_{z 0} \pm \Delta p_{z}$.

The coefficients $a, b, c, d, p_{x 0}, p_{y 0}, p_{z 0}, \Delta p_{x}, \Delta p_{y}$, and $\Delta p_{z}$ can be found in [20,21] and they are given by

$$
\begin{aligned}
& a=\left(\frac{2 \pi m}{\beta}\right)^{3 / 2}\left[g_{3 / 2}(z)-\frac{g_{5 / 2}^{2}(z)}{g_{7 / 2}(z)}\right], \quad b=c=d=\frac{1}{6}\left(\frac{2 \pi m}{\beta}\right)^{3 / 2} \frac{g_{5 / 2}^{2}(z)}{g_{7 / 2}(z)}, \\
& \Delta p_{x}=\Delta p_{y}=\Delta p_{z}=\sqrt{\frac{3 m}{\beta} \frac{g_{7 / 2}(z)}{g_{5 / 2}(z)}} \\
& p_{x o}=m u_{x}, \quad p_{y o}=m u_{y}, \quad p_{z 0}=m u_{z} .
\end{aligned}
$$

The conservative quantities carried by each beam in cell $(i, j, k)$ are $Q_{\sigma, i, j, k}=\left(R_{\sigma}, L_{\sigma}, M_{\sigma}, N_{\sigma}, E_{\sigma}\right)_{i, j, k}$, with

$$
\begin{aligned}
R_{\sigma, i, j, k} & =\int \frac{\mathrm{d} \vec{p}}{h^{3}} c_{\sigma, i, j, k} \delta\left(p_{x}-\bar{p}_{x, \sigma}, p_{y}-\bar{p}_{y, \sigma}, p_{z}-\bar{p}_{z, \sigma}\right), \\
M_{\sigma, i, j, k}^{\alpha} & =\int \frac{\mathrm{d} \vec{p}}{h^{3}} c_{\sigma, i, j, k} \frac{p_{\alpha}}{m} \delta\left(p_{x}-\bar{p}_{x, \sigma}, p_{y}-p_{0 y}, p_{z}-\bar{p}_{z, \sigma}\right), \quad \alpha=x, y, z, \\
E_{\sigma, i, j, k} & =\int \frac{\mathrm{d} \vec{p}}{h^{3}} c_{\sigma, i, j, k} \frac{p^{2}}{2 m} \delta\left(p_{x}-\bar{p}_{x, \sigma}, p_{y}-\bar{p}_{y, \sigma}, p_{z}-\bar{p}_{z, \sigma}\right),
\end{aligned}
$$


where $\bar{p}_{x, \sigma}=p_{0 x}$, for $\sigma=1,4,5,6,7, \bar{p}_{x, 2}=p_{0 x}-\Delta p_{x}$, and $\bar{p}_{x, 3}=p_{0 x}+\Delta p_{x}, \bar{p}_{y, \sigma}=p_{0 y}$, for $\sigma=1,2,3,6,7, \bar{p}_{y, 4}=$ $p_{0 y}-\Delta p_{y}$, and $\bar{p}_{y, 5}=p_{0 y}+\Delta p_{y}$ and $\bar{p}_{z, \sigma}=p_{0 y}$, for $\sigma=1,2,3,4,5, \bar{p}_{z, 6}=p_{0 z}-\Delta p_{z}$, and $\bar{p}_{z, 7}=p_{0 z}+\Delta p_{z}$. We have $c_{\sigma, i, j, k}=a_{i, j, k}$, if $\sigma=1$, and $c_{\sigma, i, j}=b_{i, j}$, if $\sigma=2,3,4,5,6,7$.

The beam velocities in the $x$-direction in cell $(i, j, k)$ are

$$
V_{2}^{x}=u_{x}+\Delta p_{x} / m, \quad V_{3}^{x}=u_{x}-\Delta p_{x} / m, \quad V_{1}^{x}=V_{4}^{x}=V_{5}^{x}=V_{6}^{x}=V_{7}^{x}=u_{x}
$$

and similarly the beam velocities in the $y$ - and $z$-direction in cell $(i, j, k)$ are

$$
\begin{aligned}
& V_{1}^{y}=V_{2}^{y}=V_{3}^{y}=V_{6}^{y}=V_{7}^{y}=u_{y}, \quad V_{4}^{y}=u_{y}+\Delta p_{y} / m, \quad V_{5}^{y}=u_{y}-\Delta p_{y} / m, \\
& V_{1}^{z}=V_{2}^{z}=V_{3}^{z}=V_{4}^{z}=V_{5}^{z}=u_{z}, \quad V_{6}^{z}=u_{z}+\Delta p_{z} / m, \quad V_{7}^{z}=u_{z}-\Delta p_{z} / m .
\end{aligned}
$$

The beam mass associated with the beam velocities are

$$
m_{1, i, j, k}=a_{i, j, k} n_{i, j, k} \Delta \hat{V}_{i, j, k}, \quad m_{\sigma, i, j, k}=b_{i, j, k} n_{i, j, k} \Delta \hat{V}_{i, j, k}, \quad \sigma=2,3,4,5,6,7,
$$

where $\Delta \hat{V}_{i, j, k}$ is the volume of cell $(i, j, k)$. The conservative quantities carried by each beam in cell $(i, j)$ are

$$
Q_{\sigma, i, j, k}=\left[\begin{array}{c}
m_{\sigma, i, j, k} \\
m_{\sigma, i, j, k} V_{\sigma, i, j, k}^{x} \\
m_{\sigma, i, j, k} V_{\sigma, i, j, k}^{y} \\
m_{\sigma, i, j, k} V_{\sigma, i, j, k}^{z} \\
\frac{1}{2} m_{\sigma, i, j, k}\left(V_{\sigma, i, j, k}^{2}+V_{\sigma, i, j, k}^{y}+V_{\sigma, i, j, k}^{z}\right)+\frac{3 g_{5 / 2}(z)}{2 \beta \lambda^{3}}
\end{array}\right]
$$

and the overall conservative quantities of gases in cell $(i, j, k)$ are defined as $Q_{i, j, k}=\sum_{\sigma=1}^{7} Q_{\sigma, i, j, k}$.

The kinetic beam formulation in two space dimensions in the Cartesian coordinates $(x, y, z)$ can be given by

$$
\partial_{t} Q_{\sigma}+\partial_{x}\left(F_{\sigma}^{+}+F_{\sigma}^{-}\right)+\partial_{y}\left(G_{\sigma}^{+}+G_{\sigma}^{-}\right)+\partial_{y}\left(H_{\sigma}^{+}+H_{\sigma}^{-}\right)=0,
$$

where the split beam fluxes $F_{\sigma}^{ \pm}$and $G_{\sigma}^{ \pm}$and $H_{\sigma}^{ \pm}$at cell $(i, j, k)$ are defined by

$$
F_{\sigma, i, j, k}^{ \pm}=V_{\sigma, i, j, k}^{x \pm} Q_{\sigma, i, j, k}, \quad G_{\sigma, i, j, k}^{ \pm}=V_{\sigma, i, j, k}^{y \pm} Q_{\sigma, i, j, k}, \quad H_{\sigma, i, j, k}^{ \pm}=V_{\sigma, i, j, k}^{z \pm} Q_{\sigma, i, j, k},
$$

where $V_{\sigma}^{\alpha \pm}=\left(V_{\sigma}^{\alpha} \pm\left|V_{\sigma}^{\alpha}\right|\right) / 2$ for $\alpha=x, y, z$.

The 3D governing equations in generalized coordinates $(\xi, \eta, \zeta)$ based on the beam splitting method can be expressed as:

$$
\partial_{t} \hat{Q}_{\sigma}+\partial_{\xi}\left(\hat{F}_{\sigma}^{+}+\hat{F}_{\sigma}^{-}\right)+\partial_{\eta}\left(\hat{G}_{\sigma}^{+}+\hat{G}_{\sigma}^{-}\right)+\partial_{\zeta}\left(\hat{H}_{\sigma}^{+}+\hat{H}_{\sigma}^{-}\right)=0,
$$

where

$$
\begin{aligned}
& \hat{Q}_{\sigma}=Q_{\sigma} / J, \quad \hat{F}_{\sigma}^{ \pm}=\left(\xi_{x} F_{\sigma}^{ \pm}+\xi_{y} G_{\sigma}^{ \pm}+\xi_{z} H_{\sigma}^{ \pm}\right) / J, \\
& \hat{G}^{ \pm}=\left(\eta_{x} F_{\sigma}^{ \pm}+\eta_{y} G_{\sigma}^{ \pm}+\eta_{z} H_{\sigma}^{ \pm}\right) / J, \quad \hat{H}_{\sigma}^{ \pm}=\left(\zeta_{x} F_{\sigma}^{ \pm}+\zeta_{y} G_{\sigma}^{ \pm}+\zeta_{z} H_{\sigma}^{ \pm}\right) / J,
\end{aligned}
$$

and $J$ is the metric Jacobian.

\section{References}

[1] S. Chapman, T.G. Cowling, The Mathematical Theory of Non-uniform Gases, Cambridge University Press, Cambridge, 1970.

[2] L.P. Kadanoff, G. Baym, Quantum Statistical Mechanics, Benjamin, New York, 1962 (Chapter 6).

[3] E.A. Uehling, G.E. Uhlenbeck, Transport phenomena in Einstein-Bose and Fermi-Dirac gases. I, Phys. Rev. 43 (1933) 552.

[4] A. Griffin, W.C. Wu, S. Stringari, Phys. Rev. Lett. 79 (1997) 553.

[5] T. Nikumi, A. Griffin, Hydrodynamic damping in trapped Bose gases, 1998, cond-mat/9711036.

[6] C. Hirsch, Numerical Computation of Internal and External Flows, vols. I \& II, Wiley, New York, 1988.

[7] E.F. Toro, Riemann Solvers and Numerical Methods for Fluid Dynamics, Springer, Berlin, 1999.

[8] R.H. Sanders, K.H. Prendergast, The possible relation of the 3-kiloparsec arm to explosions in the galactic nucleus, Astrophys. J. 188 (1974) 489.

[9] D.I. Pullin, J. Comput. Phys. 34 (1980) 231-244.

[10] R.D. Reitz, J. Comput. Phys. 42 (1981) 108. 
[11] S. Deshpande, NASA-TM 01234, 1986.

[12] K.H. Prendergast, K. Xu, Numerical hydrodynamics from gas-kinetic theory, J. Comput. Phys. 109 (1993) 53.

[13] K. Xu, K.H. Prendergast, Numerical Navier-Stokes solutions from gas-kinetic theory, J. Comput. Phys. 114 (1994) 9.

[14] S.Y. Chou, D. Baganoff, Kinetic flux-vector splitting for the Navier-Stokes equations, J. Comput. Phys. 130 (1997) 217-230.

[15] T. Ohwada, On the construction of kinetic schemes, J. Comput. Phys. 177 (2002) 156-175.

[16] P.L. Bhatnagar, E.P. Gross, M. Brook, A kinetic model for collision processes in gases, I. Small amplitude processes in charged and neutral one-component system, Phys. Rev. 94 (3) (1954) 515-525.

[17] J.Y. Yang, M.H. Chen, I.N. Tsai, J.W. Chang, A kinetic beam scheme for relativistic gas dynamics, J. Comput. Phys. 136 (1997) 19.

[18] J.L. Steger, R.F. Warming, J. Comput. Phys. 40 (1981) 262.

[19] B. van Leer, Flux vector splitting for the Euler equations, ICASE 82-30, 1982.

[20] J.Y. Yang, Y.H. Shi, A kinetic beam scheme for the ideal quantum gas dynamics, Proc. R. Soc. A 462 (2006) 1553.

[21] K. Huang, Statistical Mechanics, second ed., Wiley, New York, 1987.

[22] R.K. Pathria, Statistical Mechanics, Butterworth-Heinemann, Oxford, 1996.

[23] S.R. de Groot, G.J. Hooyman, C.A. Ten Seldam, Proc. R. Soc. A (1950) 203-266.

[24] V.C. Aguilera-Navarro, M. de Llano, M.A. Solis, Bose-Einstein condensation for general dispersion relations, Eur. J. Phys. 20 (1999) 177.

[25] A. Harten, High resolution schemes for hyperbolic conservation laws, J. Comput. Phys. 49 (1983) 357.

[26] A. Harten, B. Engquist, S. Osher, S.R. Charkravathy, Uniformly high order accurate essentially non-oscillatory schemes, III, J. Comput. Phys. 71 (1987) 231.

[27] X.-D. Liu, S. Osher, T. Chan, Weighted essentially non-oscillatory schemes, J. Comput. Phys. 115 (1994) 200.

[28] G.-S. Jiang, C.W. Shu, Efficient implementation of weighted ENO schemes, J. Comput. Phys. 126 (1996) 202.

[29] A.E. Bryson, R.W.F. Gross, Diffraction of strong shocks by cone, cylinders, and spheres, J. Fluid Mech. 10 (1961) 1-16.

[30] J.Y. Yang, Y. Liu, H. Lomax, Computation of shock-wave reflection by circular cylinders, AIAA J. 25 (5) (1987) 683-689.

[31] J.Y. Yang, J.C. Huang, Rarefied flow computations using nonlinear model Boltzmann equations, J. Comput. Phys. 120 (1995) 323 339. 